\title{
Vibrio cholerae O1 clinical strains isolated in 1992 in Kolkata with progenitor traits of the 2004 Mozambique variant
}

\section{Correspondence \\ Ranjan K. Nandy \\ nandyrk@icmr.org.in}

Received 23 May 2008

Accepted 15 October 2008

\author{
Souvik Chatterjee, ${ }^{1} \dagger$ Tapas Patra, ${ }^{1} \dagger$ Kausik Ghosh, ${ }^{1}$ Amit Raychoudhuri, ${ }^{1}$ \\ Gururaja P. Pazhani, ${ }^{1}$ Mayukh Das, ${ }^{1}$ Banwarilal Sarkar, ${ }^{1}$ Rupak K. Bhadra, ${ }^{2}$ \\ Asish K. Mukhopadhyay, ${ }^{1}$ Yoshifumi Takeda, ${ }^{3}$ G. Balakrish Nair, ${ }^{1}$ \\ Thandavarayan Ramamurthy ${ }^{1}$ and Ranjan K. Nandy ${ }^{1}$ \\ ${ }^{1}$ National Institute of Cholera and Enteric Diseases, Kolkata, India \\ ${ }^{2}$ Indian Institute of Chemical Biology, Kolkata, India \\ ${ }^{3}$ Collaborative Research Center of Okayama University for Infectious Diseases, National Institute of \\ Cholera and Enteric Diseases, Kolkata, India
}

\begin{abstract}
Retrospective analysis led to the detection of two Vibrio cholerae variant O1 strains (VC51 and VC53), which were isolated in 1992 in Kolkata from clinical cases, with identical traits to 2004 Mozambique variant $\mathrm{O} 1$ strains. The Mozambique $\mathrm{O} 1$ strains that caused a huge outbreak in 2004 have been shown to have phenotypic traits of both classical and El Tor biotypes, and thereby have been reported as variant. Our study demonstrated that two $\mathrm{O} 1$ strains isolated in Kolkata during 1992 were of the El Tor background as evidenced by polymyxin $\mathrm{B}\left(50 \mathrm{U} \mathrm{ml}^{-1}\right)$ resistance, positivity in Voges-Proskauer reactions and sensitivity to biotype-specific vibrio phages. With the features of classical CTX prophage, localization in the small chromosome, and an absence of RS1 and pTLC, both Mozambique and Kolkata strains appeared to be identical. Furthermore, two Kolkata strains exhibited an identical ribotype to that of the Mozambique variant, displaying ribotype pattern $\mathrm{RI}$ that had been assigned to Kolkata V. cholerae $\mathrm{O} 1$ strains isolated on or before 1992. Notl pulsotype analysis indicated that these 1992 Kolkata strains along with the Mozambique variant $\mathrm{O} 1$ belonged to very closely related clones. Considering the chronological events, and the typical identity at the phenotypic and the genotypic level between the two O1 strains isolated during 1992 from Kolkata and during 2004 from Mozambique, we propose that some of the 1992 Kolkata O1 strains might have acted as progenitors for Mozambique variant $\mathrm{O} 1$ strains.
\end{abstract}

\section{INTRODUCTION}

Vibrio cholerae $\mathrm{O} 1$ and $\mathrm{O} 139$ strains are responsible for causing the disease cholera that afflicts million of people every year. To date, human civilization has witnessed seven pandemics of cholera and the seventh pandemic is still ongoing from its beginning in 1961 (Kaper et al., 1995). The members of the serogroup O1 are classified into two biotypes, classical and El Tor, and two major serotypes, Ogawa and Inaba (Kaper et al., 1995). While immunological specificity of the 'O' antigenic polysaccharides forms the basis of serotype differentiation, biotype differentiation is based on the phenotypic traits that include the typical resistance phenotype of the El Tor

†These authors contributed equally to this work.

Abbreviations: $\mathrm{CHEF}$, contour-clamped homogeneous electric field; NICED, National Institute of Cholera and Enteric Diseases. strains against $50 \mathrm{U}$ polymyxin B, positivity for VogesProskauer reactions, agglutination with chicken erythrocytes, haemolysis of sheep erythrocytes and sensitivity to El Tor group V vibrio phages (Kaper et al., 1995). However, the test for the lysis of sheep erythrocytes is of limited use as most of the El Tor strains after the 1960s became nonhaemolytic (Barrett \& Blake, 1981). In addition to conventional biotyping methods, nowadays, molecular methods are widely in use that target biotype-specific allelic gene loci. These include biotype-specific alleles of tcpA and CTX prophages (Davis et al., 1999; Keasler \& Hall, 1993).

The aetiological agent of the seventh cholera pandemic is $V$. cholerae O1 biotype El Tor, which has replaced classical biotype strains over a period of time. In a recent study, strains with hybrid biotype traits (Matlab variants) have been reported from Bangladesh (Nair et al., 2002). These 
Matlab variants were negative for the Voges-Proskauer reaction, a trait typical for the classical biotype. Interestingly, these strains were resistant to lysis by the classical-biotype-specific vibrio phages. In fact, these strains displayed phenotypes that were not specific for either of the two biotypes and therefore they were considered as hybrids. The latest addition is the emergence and progression of a unique variant of $V$. cholerae $\mathrm{O} 1$ from Mozambique in 2004 (Ansaruzzaman et al., 2004, 2007). The Mozambique O1 strains have been reported to possess traits of both classical and El Tor biotypes. In contrast to the Matlab variants, the background of Mozambique variants is typical El Tor as it displayed resistance to classical vibrio phages and polymyxin $\mathrm{B}\left(50 \mathrm{U} \mathrm{ml}^{-1}\right)$, but was sensitive to El Tor group $\mathrm{V}$ vibrio phages and positive for Voges-Proskauer reactions. All these traits still remain as the most reliable biotype differentiation markers for $V$. cholerae O1. The classical biotype properties of the Mozambique variant included detection of classical CTX prophage $\left(\mathrm{CTX}^{\mathrm{Cl}}\right)$ localized in the small chromosome and absence of RS1 (Das et al., 2007; Faruque et al., 2007). In addition, these strains were also devoid of the toxin linked cryptic plasmid (pTLC) (Rubin et al., 1998). Comparative analysis of the PFGE profiles of Matlab and Mozambique variants revealed that these two groups of strains belonged to different clones (Ansaruzzaman et al., 2007).

The identification and rapid spread of the Mozambique variant led researchers to consider whether there had been an emergence of a more virulent variant of the El Tor biotype that possibly had the added advantages of the El Tor background with virulence phenotypes of classical origin. Previously, we have reported on the variant traits of $V$. cholerae $\mathrm{O} 1 \mathrm{El}$ Tor strain VC20 that possessed both classical and El Tor alleles of $r s t R$, one of the constituent genes of CTX prophage (Bhattacharya et al., 2006); the strain was isolated in 1992 from clinical cases. Detection of such a variant prompted us to revive archived $V$. cholerae O1 strains to screen them for traits similar to that of the Mozambique variant isolated previously. Detailed characterization of 15 O1 strains isolated during 1992 in Kolkata led to the identification of two strains with progenitor traits of the 2004 Mozambique variant.

\section{METHODS}

Bacterial strains. Clinical $V$. cholerae O1 strains isolated during 1992 were revived from cultures maintained at the National Institute of Cholera and Enteric Diseases (NICED), Kolkata. The bacterial strains were maintained in nutrient agar stabs and stored in the dark. Fifteen V. cholerae O1 strains including VC20 (Bhattacharya et al., 2006), were considered in this study. Reference V. cholerae O1 strains of classical (569B, O395) and El Tor (N16961) biotypes were included as a comparison. The $\mathrm{O} 1$ variant strain $\mathrm{B} 33$ isolated from the Mozambique outbreak was also included (Ansaruzzaman et al., 2004).

Detection of biotype traits. Biotype-specific traits were assayed by determining the susceptibilities to polymyxin B and to biotypespecific vibrio phages. In addition, Voges-Proskauer reaction patterns were also determined for $V$. cholerae strains by growing the cells in
MR-VP (methyl red-Voges-Proskauer) medium supplemented with $1 \% \mathrm{NaCl}$ (Kelly et al., 1992). Susceptibility to polymyxin B was determined by plate assay using Mueller-Hinton (MH) agar containing $50 \mathrm{U}$ polymyxin $\mathrm{B} \mathrm{ml}^{-1}$ (Sigma). For this, cells were grown in $5 \mathrm{ml} \mathrm{MH}$ broth for $4-5 \mathrm{~h}\left(\mathrm{OD}_{540} \sim 0.3\right)$ at $37^{\circ} \mathrm{C}$ and $10 \mu \mathrm{l}$ culture was carefully placed on an $\mathrm{MH} /$ polymyxin $\mathrm{B}\left(50 \mathrm{U} \mathrm{ml}^{-1}\right.$ ) plate and allowed $30 \mathrm{~min}$ for absorption. The polymyxin $\mathrm{B}$ resistance phenotype was assigned to those strains that grew on the plate after $18 \mathrm{~h}$ incubation at $37^{\circ} \mathrm{C}$. Susceptibility patterns of these strains towards El Tor group $\mathrm{V}$ or classical group IV phages were determined (Chakrabarti et al., 2000). Briefly, 3-4 h statically grown $V$. cholerae cells were mixed with molten $\left(\sim 45{ }^{\circ} \mathrm{C}\right) 0.8 \%$ soft agar, poured onto a nutrient agar plate and $20-30 \mathrm{~min}$ allowed for solidification. Next, each of the biotype-specific phages was spotted (from routine test dilutions) separately onto these plates. Next, plates were kept at room temperature for $10 \mathrm{~min}$ to allow the drops to dry. Following an incubation of $18 \mathrm{~h}$ at $37^{\circ} \mathrm{C}$, each reaction was recorded as positive if there was detection of a halo zone or five or more plaques.

PCR assay and nucleotide sequencing. Genomic DNA free from RNA and proteinaceous material was isolated from $V$. cholerae strains grown in Luria-Bertani medium at $37{ }^{\circ} \mathrm{C}$ overnight (Murray \& Thompson, 1980). Briefly, cells were harvested, washed and resuspended in TE buffer (10 mM Tris/HCl, $1 \mathrm{mM}$ EDTA), pH 8.0. Next, cells were lysed with SDS followed by digestion with proteinase $\mathrm{K}\left(20 \mu \mathrm{g} \mathrm{m}^{-1}\right)$ for $2 \mathrm{~h}$ at $60{ }^{\circ} \mathrm{C}$. Purified genomic DNA free from proteinaceous materials and RNA was obtained by treatment with $\mathrm{N}$ cetyl- $N, N, N$-trimethylammonium bromide $(10 \% \mathrm{CTAB}$ in $0.5 \mathrm{M}$ $\mathrm{NaCl})$ at $65{ }^{\circ} \mathrm{C}$ for $10 \mathrm{~min}$, and sequential extraction with phenol/ chloroform/isoamyl alcohol $(25: 24: 1$, by vol. $)$ and chloroform. Purified genomic DNA was subjected to ethanol precipitation, airdried, reconstituted in sterile water and stored at $4{ }^{\circ} \mathrm{C}$. The DNA was then used in the PCR assay to detect the $r s t R$ alleles (Bhattacharya et al., 2006). Detection of 474 or $501 \mathrm{bp}$ amplicons corresponded to the presence of $r s t R$ alleles of classical $\left(r s t R^{C l}\right)$ or El Tor $\left(r s t R^{E l}\right)$ types, respectively. Details of the PCR primers used in this study are presented in Table 1. In the multiplex assay, toxigenic traits and the presence of the biotype-specific tcpA allele among these $V$. cholerae strains were determined (Keasler \& Hall, 1993). PCR amplicon of $301 \mathrm{bp}$ corresponded to the presence of $c t x A$, while $472 \mathrm{bp}$ or $618 \mathrm{bp}$ amplicons represented the presence of the tcpA allele of El Tor or classical biotypes, respectively. Presence or absence of the RS1 element among these strains was determined using a combination of PCR primers Ig1F and RstCR. Detection of an amplicon of $2270 \mathrm{bp}$ indicated the presence of RS1 among these strains. A simplex PCR assay using the primers Tlc-F and Tlc-R generated a $2032 \mathrm{bp}$ amplicon for the pTLC positive strains. Amplification of the CII region (small chromosome) that spans either side of the CTX prophage when localized in the small chromosome was also carried out (Das et al., 2007; Maiti et al., 2006). For this, combinations of PCR primers CIIF-F/RstA-3R and CtxAF/CII-R were used. Localization of the CTX prophage in the small chromosome results in 1.8 and $1.4 \mathrm{~kb}$ amplicons in the PCR assays with CII-F/RstA-3R and CtxAF/CII-R primers, respectively. Detection of a $760 \mathrm{bp}$ amplicon in the PCR assays with CII-F/CII-R was interpreted as detection of empty site in the small chromosome (Das et al., 2007). PCR amplicons obtained for the alleles of the gene loci $r s t R$ and $t c p A$ were sequenced directly using a BigDye terminator cycle sequencing kit (Applied Biosystems) and alignment analysis made with the respective nucleotide sequences available in a public database (www.ncbi.nlm.nih.gov) to confirm their identity as respective allele. The $\operatorname{ctxB}$ gene of $\mathrm{O} 1 \mathrm{~V}$. cholerae strains belonging to classical and $\mathrm{El}$ Tor biotypes differs at nucleotide positions 115 and 203, where the first base of the start codon is considered as nucleotide 1 (Popovic et al., 1994). An amplicon of 465 bp generated through PCR assay using primers CtxBF/CtxBR (Olsvik et al., 1993) was therefore sequenced directly to determine the nature of $c t x B$ present among these strains. 
Table 1. List of PCR primers and amplicon sizes of target genes

$r s t R$, igl, rstC, $c t x A$ and $c t x B$ are the genes in the CTX prophage; $t c p A$ represents a gene in the $V$. cholerae pathogenicity island.

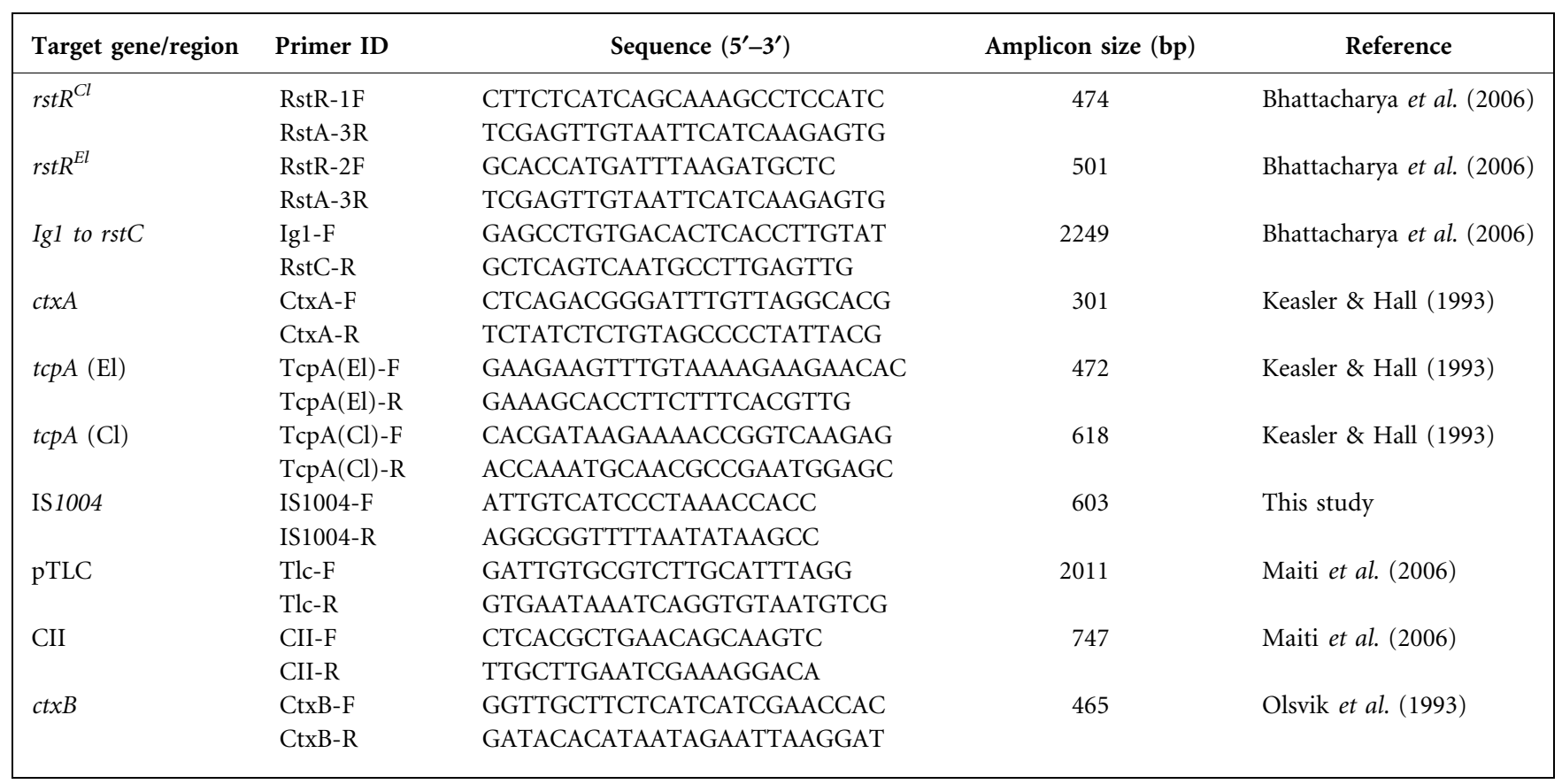

Cl, Classical allele; El, El Tor allele.

DNA probes and molecular typing. A PCR amplicon of $603 \mathrm{bp}$ generated with IS1004F/IS1004R primers from O1 strain O395 was cloned into pGEM-T EASY vector (Promega) to generate pKG506. Similarly, a $2032 \mathrm{bp}$ amplicon of pTLC generated from the O1 strain O395 was cloned into pCR2.1 (Invitrogen) to generate pSC20. The identity of the cloned fragments compared to IS1004 and pTLC in pKG506 and pSC20, respectively, was confirmed by nucleotide sequencing using M13 universal primers (Takara Shuzo). The EcoRI fragments of pKG506 and pSC20 served as specific DNA probes for IS 1004 and pTLC, respectively. The $7.5 \mathrm{~kb}$ BamHI fragment of pKK3535 served as a specific DNA probe for rDNA and was used in ribotype analysis (Brosius et al., 1981). The IS1004 typing and ribotype analysis was carried out with BglI-digested genomic DNA. The digested DNA was electrophoresed in agarose gels, transferred to nylon membrane (HybondN ${ }^{+}$; Amersham Pharmacia Biotech) and allowed to hybridize with a specific DNA probe. The hybridized membrane was developed using a non-radioactive labelling kit using the substrate CDP-Star following the procedures described by the manufacturer (Amersham Pharmacia Biotech). Results were recorded on X-ray film, which was subsequently processed digitally and analysed using a Bio-Rad documentation system. Dot-blot analysis was carried out to determine the presence or absence of pTLC among these strains. For this, $2 \mu \mathrm{l}$ purified genomic DNA $\left(50 \mathrm{ng} \mu \mathrm{l}^{-1}\right)$ was denatured and spotted onto nylon membrane (Hybond- $\mathrm{N}^{+}$) to form an array. The DNA was immobilized on the membrane and allowed to hybridize with labelled DNA probe specific to pTLC. The membrane was developed, and results were recorded on X-ray film and digitalized with the Bio-Rad documentation system.

PFGE. Agarose-embedded genomic DNA from $V$. cholerae strains was treated with $50 \mathrm{U}$ NotI (Takara) and the DNA fragments were separated electrophoretically under the contour-clamped homogeneous electric field (CHEF) method on a CHEF Mapper system (Bio$\mathrm{Rad}$ ) (Chatterjee et al., 2007). Run conditions were generated by the autoalgorithm mode of the CHEF Mapper PFGE system using a size range of 20 to $300 \mathrm{~kb}$ that was typically followed an electrophoresis time of $40 \mathrm{~h} 24 \mathrm{~min}$ with $1 \%$ PFGE grade agarose gel in $0.5 \times \mathrm{TBE}$ (44.5 mM Tris/HCl, $44.5 \mathrm{mM}$ boric acid, $1.0 \mathrm{mM}$ EDTA, $\mathrm{pH}$ 8.0) at $14{ }^{\circ} \mathrm{C}$. Following electrophoresis, the gels were stained for $30 \mathrm{~min}$ in Elix MilliQ water (Millipore) containing $1.0 \mu \mathrm{g}$ ethidium bromide $\mathrm{ml}^{-1}$, destained in Elix MilliQ water for $15 \mathrm{~min}$ and photographed under UV light using the Gel Doc 2000 gel documentation system (Bio-Rad).

\section{RESULTS AND DISCUSSION}

\section{Classical CTX prophage in the 1992 Kolkata 01 strains VC51 and VC53}

$V$. cholerae O1 strains that were isolated in 1992 from clinical cases and archived in the repository maintained by NICED were revived and the identity of these strains was reconfirmed by conventional microbiological techniques: serogroup analysis through slide agglutination tests using antisera specific to the O1 serogroup followed by serotyping with antisera specific to Ogawa and Inaba serotypes (Denka Seiken). An analysis was carried out to detect strains with genotypic traits resembling classical biotype of $V$. cholerae O1. These traits included the presence of biotype-specific alleles of CTX prophages and the $t c p A$ gene that encodes a major subunit protein of toxin co-regulated pilus (TCP) (Davis et al., 1999; Keasler \& Hall, 1993). Among the constituent genes of the CTX prophages, heterogeneity at the nucleotide level of $r s t R$, the phage 
repressor located on the RS2 region of CTX prophage, formed the basis of differentiation between CTX prophages of classical $\left(\mathrm{CTX}^{\mathrm{Cl}}\right)$ and $\mathrm{El}$ Tor $\left(\mathrm{CTX}^{\mathrm{El}}\right)$ types (Davis et al., 1999; Kimsey \& Waldor, 1998). A PCR-based assay has been developed by exploiting the variations that exist between the rstR loci of the $\mathrm{CTX}^{\mathrm{Cl}}$ and $\mathrm{CTX}^{\mathrm{El}}$ phages (Bhattacharya et al., 2006). PCR-based screening was carried out using allele-specific $r s t R$ against clinical $V$. cholerae strains isolated in 1992 in Kolkata. Analysis revealed only two strains, VC51 and VC53, that produced the $474 \mathrm{bp}$ amplicon specific to $r s t R^{C l}$ but failed to generate the $501 \mathrm{bp}$ amplicon specific for $r s t R^{E l}$ (Fig. 1a, b; lanes 1, $2)$. Excepting these two strains, none of the remaining strains $(n=13)$ produced only $r s t R^{C l}$ amplicon. However, six strains gave amplicons specific to both $r s t R^{C l}$ and $r s t R^{E l}$ along with the O1 strain VC20. Results generated with representative strains are presented in Fig. 1. The 1992 O1 strains VC49 (Fig. 1, lane 3) and VC20 (Fig. 1, lane 6) possessed both alleles of $r s t R$ as they produced amplicons of $474 \mathrm{bp}$ and $501 \mathrm{bp}$ when assayed under identical conditions. The reference $\mathrm{O} 1$ variant strain isolated from the Mozambique outbreak (B33) produced only $r s t R^{C l}$ (Fig. 1; lane 5). The reference strains of O1 El Tor (N16961) (Fig. 1, lane 7) and classical (O395, 569B) (Fig. 1, lanes 8,9$)$ biotypes gave $r s t R$-specific amplicons for the
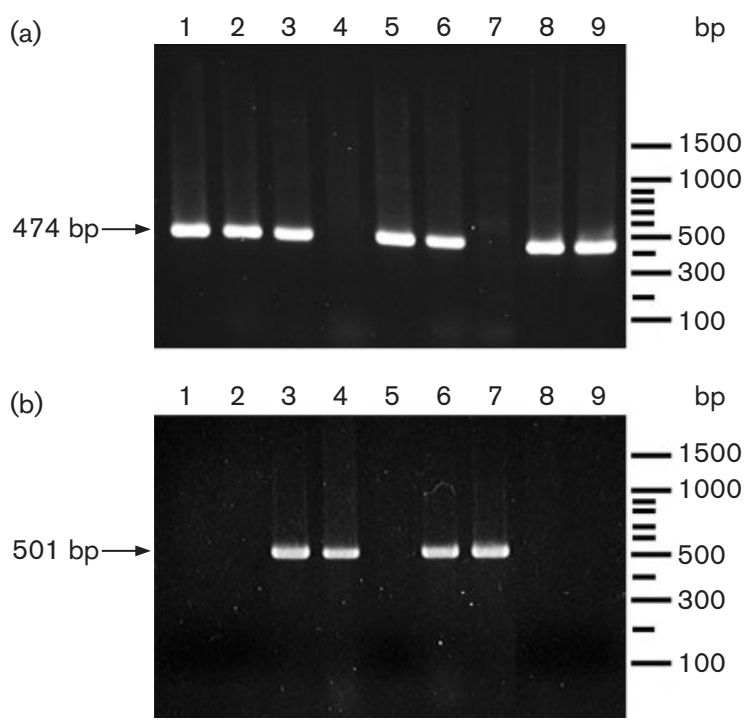

Fig. 1. Agarose gel electrophoresis patterns of PCR amplicons obtained with primers specific to rstR of classical $\left(r s t R^{C l}\right)$ (a) and El Tor $\left(r s t R^{E I}\right)(b)$ types with $V$. cholerae strains. The V. cholerae strains included were isolated in Kolkata during 1992 (lanes: 1, VC51; 2, VC53; 3, VC49; 4, VC61; 6, VC20). Reference V. cholerae O1 strains biotype El Tor (lane 7, N16961) and classical (lanes 8, O395; lane 9, 569B) and 2004 Mozambique O1 variant (lane 5, B33) were included as controls. Amplicon sizes of 474 and $501 \mathrm{bp}$ specific for $r s t R^{C l}$ and $r s t R^{E I}$, respectively, are indicated. The band positions of the $100 \mathrm{bp}$ ladder (Takara) run on the same gel are also indicated. respective biotypes. The $r s t R$-based screening was also carried out with clinical $V$. cholerae strains that were isolated between 1989-1991 and 1993-1994, at least 10 strains were randomly selected for each of the 5 years. However, none of these strains produced only an $r s t R^{C l}$-specific amplicon (data not shown). Excepting the strains VC51 and VC53, all other strains showed either both types of $r s t R$ alleles $\left(r s t R^{C l}\right.$ and $r s t R^{E l}$ ) or only the El Tor allele $\left(r s t R^{E l}\right)$. These data indicated that the 1992 Kolkata O1 strains VC51 and VC53 possessed only classical CTX prophage. The results were extended further by determining the nucleotide sequence of the $r s t R$. A PCR amplicon spanning the entire $r s t R$ region was generated from 1992 Kolkata O1 strains VC51 and VC53, and sequenced directly. Nucleotide sequence analysis revealed complete identity of the $r s t R$ gene to that of the classical allele (GenBank accession no. AF055890) and to the Mozambique variant strain B33 as well. These data corroborated well with the PCR-based detection of $r s t R^{C l}$ alone (Fig. 1a). The presence of classical CTX prophage in the 1992 Kolkata O1 strains (VC51 and VC53) was further confirmed by the analysis of $c t x B$ locus. Alignment analysis of the $c t x B$ nucleotide sequences derived from the 1992 Kolkata O1 strains revealed complete identity among themselves and to the $c t x B$ gene of strain B33 (GenBank accession no. EF158842), as well as the reported sequence of a classical O1 strain 569B (GenBank accession no. U25679).

\section{Small chromosomal localization of the classical CTX prophage in the 1992 Kolkata 01 strains (VC51 and VC53) that resembled the Mozambique variant}

The Mozambique variant $\mathrm{O} 1$ strains were reported as unique in that the CTX prophage genome was classical while the background of the strain remained typical El Tor (Ansaruzzaman et al., 2007; Das et al., 2007; Faruque et al., 2007). The filamentous phage was reported to be present in the small chromosome, a trait similar to that of the classical biotype. Detection of classical CTX prophage in the 1992 Kolkata O1 strains prompted us to characterize its chromosomal localization. For this, PCR-based analysis was carried out using primers specific to the region (CII) that spans either side of the CTX prophage localized in the small chromosome (Maiti et al., 2006). The PCR assay was carried out with primer combination of CII-F/RstA-3R and CtxAF/CII-R (Maiti et al., 2006). Data on representative strains are presented in Fig. 2. It is evident from Fig. 2 that 1992 Kolkata O1 strains (VC51, VC53) (lanes 1, 2) as well as the Mozambique O1 variant strain B33 (lane 5), produced amplicons of 1.8 and $1.4 \mathrm{~kb}$ in size for CII-F/ RstA-3R and CtxAF/CII-R PCR assays, respectively. Identical amplicons were also obtained with reference $\mathrm{O} 1$ classical strains O395 and 569B (lanes 8, 9). The reference O1 El Tor strain N16961 (lane 7) failed to generate any band due to the absence of the CTX prophage in the small chromosome. The presence of an empty CTX prophage site in the small chromosome of N16961 was further confirmed by the detection of a $747 \mathrm{bp}$ amplicon generated with 

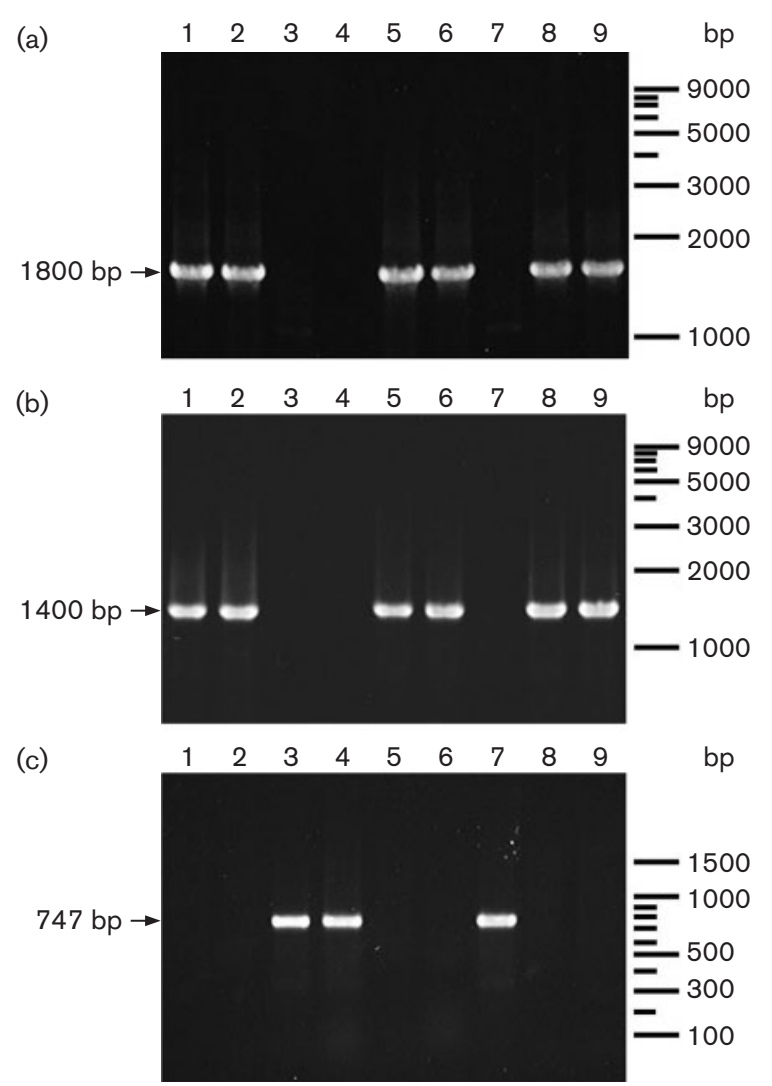

Fig. 2. Agarose gel electrophoresis patterns of $P C R$ amplicons obtained with primers Cll-F/RstA-3R (a), CtxA-F/CII-R (b) and CIIF/CII-R (c) with V. cholerae strains. The V. cholerae strains included were isolated in Kolkata during 1992 (lanes: 1, VC51; 2, VC53; 3, VC49; 4, VC61; 6, VC20). Reference V. cholerae O1 strains biotype El Tor (lane 7, N16961) and classical (lanes 8, O395; 9, 569B) and 2004 Mozambique O1 variant (lane 5, B33) were included as controls. Amplicon sizes of $1.8 \mathrm{~kb}, 1.4 \mathrm{~kb}$ and $747 \mathrm{bp}$ specific for Cll-F/RstA-3R, CtxA-F/CII-R and Cll-F/CII-R, respectively, are indicated. The band positions of the $1 \mathrm{~kb}(\mathrm{a}, \mathrm{b})$ and $100 \mathrm{bp}$ (c) ladders (Takara) run on the same gel are also indicated.

CII-F/CII-R primers (Fig. 3c, lane 7). The 1992 O1 strains VC51 and VC53, along with B33 and classical strains $(\mathrm{O} 395,569 \mathrm{~B})$, failed to give any band in the PCR assay with CII-F/CII-R due to the presence of CTX prophage in this region. Detection of classical CTX prophage and its localization in the small chromosome of 1992 O1 strains is a testimony of the close similarity between the 1992 Kolkata O1 strains (VC51 and VC53) to that of the Mozambique O1 variant.

\section{El Tor biotype traits of Kolkata 01 strains and genotypic identity to Mozambique 01 variant}

The 1992 Kolkata O1 strains VC51 and VC53 displayed typical identity to the Mozambique variant $\mathrm{O} 1$ strain $\mathrm{B} 33$ as these strains possessed classical CTX prophage localized
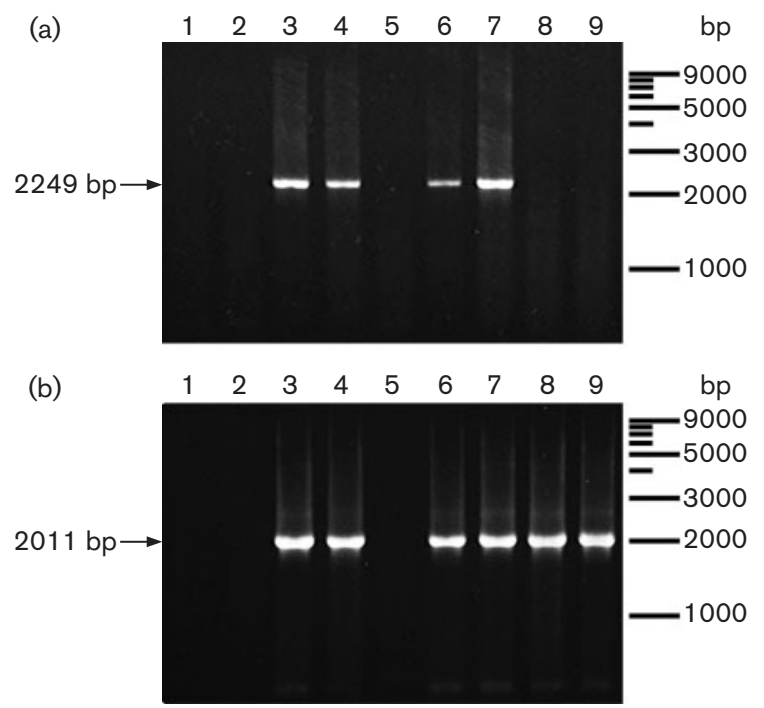

Fig. 3. Agarose gel electrophoresis patterns of PCR amplicons obtained with primers Ig1-F/RstC-R (a) and pTLC (b) with $V$. cholerae strains. The $V$. cholerae strains included were isolated in Kolkata during 1992 (lanes: 1, VC51; 2, VC53; 3, VC49; 4, VC61; 6 , VC20). Reference V. cholerae O1 strains biotype El Tor (lane 7, N16961) and classical (lanes: 8, O395; 9, 569B) and 2004 Mozambique $\mathrm{O} 1$ variant (lane 5, B33) were included as controls. Amplicon sizes of 2249 bp and 2011 bp specific for the region spanning ig $1 F$ to $r s t C$ and $p T L C$, respectively, are indicated. The band positions of the $1 \mathrm{~kb}$ ladder (Takara) run on the same gel are also indicated.

in the small chromosome, a trait similar to the classical biotype but not the El Tor biotype. Therefore, detailed analysis was carried out to help elucidate the biotype background of the 1992 Kolkata O1 strains VC51 and VC53. Analysis revealed that these strains were resistant against $50 \mathrm{U}$ polymyxin $\mathrm{B}$, a stable phenotype typically associated with the El Tor biotype. Although the 1992 O1 strains (VC51, VC53) displayed the classical trait of the presence of the classical CTX prophage $\left(r s t R^{C l}, c t \times B^{C l}\right)$, the background of these strains was considered as El Tor on the basis of their polymyxin B resistance phenotype. The lineage of the El Tor biotype for these strains was further confirmed through positive reactions to the VogesProskauer test and their sensitivity to El Tor group V vibrio phage (Table 2). The strain B33 displayed an identical phenotype to that of these two 1992 strains (Table 2). The analysis was extended further to reveal presence or absence of genes that were reported to act as markers for the detection/ differentiation of Matlab and Mozambique variants of $V$. cholerae O1 (Ansaruzzaman et al., 2007; Safa et al., 2006). Multiplex-PCR-based assay revealed that two 1992 strains (VC51 and VC53) possessed both $\operatorname{ctxA}$ and $t c p A$ (Table 2). The tcpA of these two strains was El Tor type, a phenotype common for 2004 Mozambique variant but not for the polymyxin B-resistant Matlab hybrid that was identified in Bangladesh 


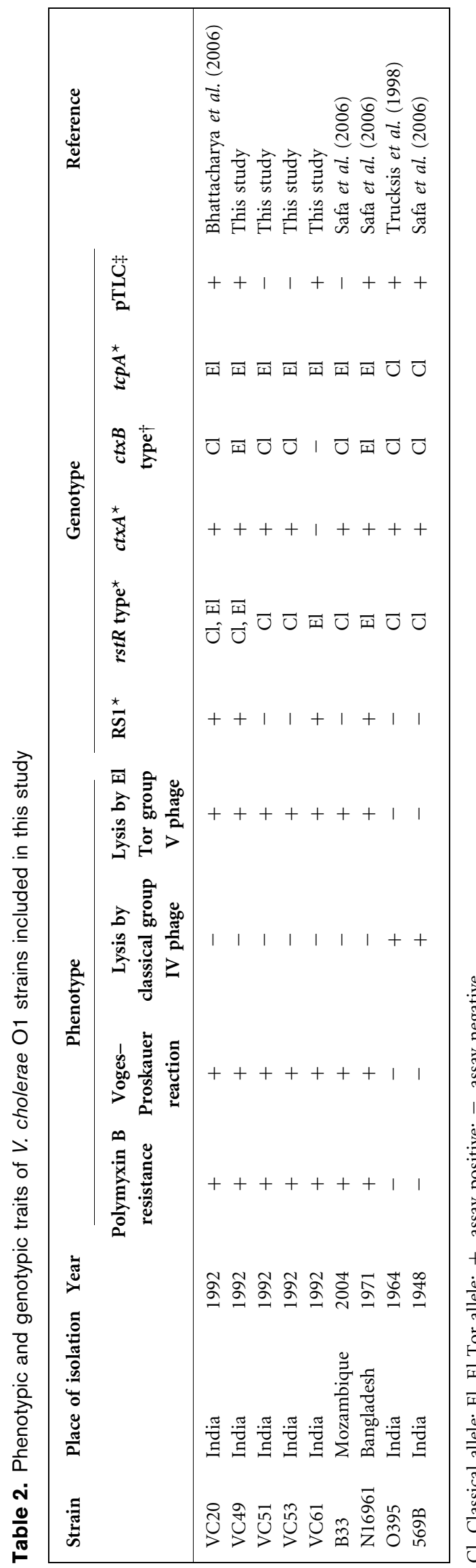

(Ansaruzzaman et al., 2007; Nair et al., 2002). Considering the striking similarity to that of the Mozambique variant O1 strain, these 1992 strains (VC51 and VC53) were analysed for the detection of RS1 and pTLC. Detection of RS1 was carried out by PCR assay using the primers Ig1F and RstCR (Table 1). The reference O1 El Tor strain N16961 gave an amplicon of $\sim 2.2 \mathrm{~kb}$ in size (Fig. 3a, lane 7), while the two $1992 \mathrm{O} 1$ strains (VC51 and VC53) failed to generate any amplicons (Fig. 3a, lanes 1,2). The PCR negativity was also evident against Mozambique variant strain B33 and reference O1 classical strains 569B and O395 (Fig. 3a, lanes 5, 8, 9). Excepting the strains VC51 and VC53, other strains that were also isolated in 1992 (VC49, VC61 and VC20) gave a positive amplicon of $\sim 2.2 \mathrm{~kb}$ under identical conditions and thereby confirmed the presence of RS1 among these strains (Fig. 3a, lanes 3, 4, 6). The RS1 element has been shown to be linked with the CTX prophage of $V$. cholerae O1 El Tor, and O139 strains in general, but existence of free RS1 in $V$. cholerae is not rare. Interestingly, the $\mathrm{O} 1$ classical strains are known to be devoid of RS1 (Davis et al., 2000). The absence of RS1 among the 1992 Kolkata O1 strains (VC51 and VC53) pointed towards the existence of a genotype known for the classical biotype despite the fact that these strains displayed certain stable El Tor phenotypes. Similar phenotypes were reported for the 2004 Mozambique variant O1 strains B33 (Faruque et al., 2007). The identity at the genetic level between the 1992 Kolkata variant O1 strains (VC51 and VC53) and 2004 Mozambique variant O1 strain B33 was further confirmed through PCR negativity with primers specific to pTLC (Fig. 3b, lanes 1, 2, 5) and the data were further substantiated through dot-blot analysis with a DNA probe specific to pTLC. No recognition by the PTLC probe was evident against 1992 Kolkata strains (VC51 and VC53) or B33. Excepting these three, reference $V$. cholerae O1 El Tor and classical biotypes strains gave the expected amplicon and were recognized by the pTLC probe. Therefore, the strains VC51 and VC53 displayed traits that are known to be unique for Mozambique variant $\mathrm{O} 1$ strain B33.

\section{Macro restriction analysis of Kolkata and Mozambique variant strains}

DNA fingerprinting analysis has been widely used to demonstrate clonal relationships among bacterial strains. The RFLP associated with the presence of insertion (IS) elements has been used successfully for the differentiation of the strains of a variety of bacterial species. An IS element, IS1004, was identified among $V$. cholerae strains of O1 and O139 origin (Bik et al., 1996) and it was successfully used to demonstrate that $\mathrm{O} 139 \mathrm{~V}$. cholerae evolved from O1 El Tor strains. Southern hybridization analysis, carried out with BglI-digested genomic DNA and DNA probes specific to IS1004, revealed identical patterns among the El Tor background strains that diverged from classical strain. Representative profiles generated with $V$. cholerae strains are presented in Fig. 4(a). It is evident that 
(a)

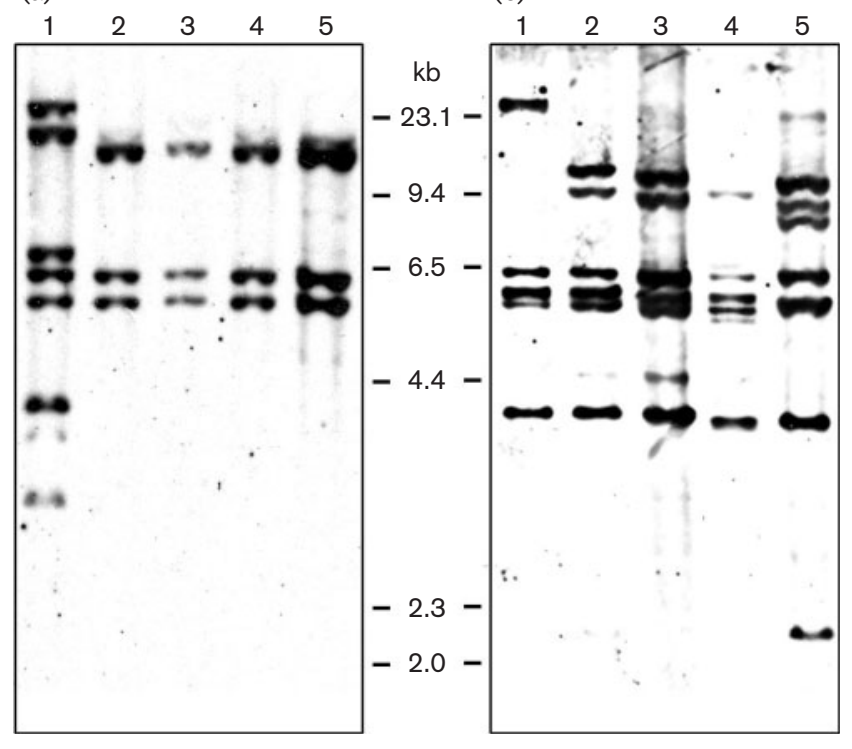

Fig. 4. The Bgll IS1004 typing (a) and ribotype (b) patterns obtained for $V$. cholerae strains. The $V$. cholerae $\mathrm{O} 1$ strains included were classical 569B (lane 1), 1992 Kolkata variant VC51 (lane 2), 2004 Mozambique variant B33 (lane 3), El Tor reference strain N16961 (lane 4) and 2003 Kolkata strain CO366 (lane 5). The band positions of the $\lambda$-Hindlll markers run on the same gel are also indicated.

1992 Kolkata O1 strains (lanes 2) displayed a profile identical to that of the reference O1 El Tor strain N16961 (lane 4) and Mozambique variant O1 strain B33 (lane 3). However, the profile obtained with classical strain 569B (lane 1) was very different. These data confirmed the lineage of the 1992 Kolkata O1 strains to be that of the El Tor biotype at the genetic level. The analysis was extended further by generating ribotype profiles for these strains, a technique that has been used successfully to demonstrate the emergence and progression of different clones of $V$. cholerae strains that have been circulating in the Indian subcontinent over a period of time. It has already been reported that $V$. cholerae $\mathrm{O} 1$ strains that were isolated before the O139 outbreak in late 1992 had a typical BglI ribotype profile named RI (Sharma et al., 1997). The RII and RIII BglI ribotype profiles were assigned to $V$. cholerae O1 strains isolated during and after the O139 outbreaks. As expected, the 1992 Kolkata O1 strains displayed a profile identical to that of RI, which was identical to that of the 2004 Mozambique variant O1 strain B33 (Fig. 4b, lanes 2, 3) but differed from that of the classical strain 569B (Fig. 4b, lane 1). The RI profile obtained with the 1992 Kolkata O1 strain had diverged from the profile obtained with O1 El Tor strains N16961 as well as reference O1 strain for RIII profile CO366 (Sharma et al., 1997). Apart from ribotyping, PFGE analysis is also a very useful technique to illustrate the clonal relatedness among different strains of the same species. NotI pulsotype patterns were reported to have the most discriminatory power when applied to $V$. cholerae strains. The NotI pulsotype patterns of the 1992 Kolkata O1 strains (VC51 and VC53) with hybrid traits of classical and El Tor biotypes were generated and compared to the pattern of B33. It is evident that the two $\mathrm{O} 1$ strains of Kolkata differed from each other by two bands of sizes $\sim 80$ and $\sim 100 \mathrm{~kb}$ (Fig. 5, lanes 2, 3). Though both the strains were isolated in Kolkata during 1992, variations in the profile indicated that these two strains did not belong to a single clone but belonged to closely related clones. Comparative analysis also showed variations with 2004 Mozambique variant O1 strains. The 1992 Kolkata variant O1 strains (VC51 and VC53), and the 2004 Mozambique variant O1 strain B33, displayed almost identical phenotypes and genotypes excepting pulsotype profile. Considering chronological events, we hypothesized that the 1992 Kolkata O1 variant strains might have acted as precursors to the 2004 Mozambique variant O1 strains. Considering the 12 year span between the isolation of Kolkata and Mozambique variant strains, and their different geographical locations, it is quite possible that strains had undergone changes at the genetic level that are only evident through PFGE analysis and not with other molecular tools.

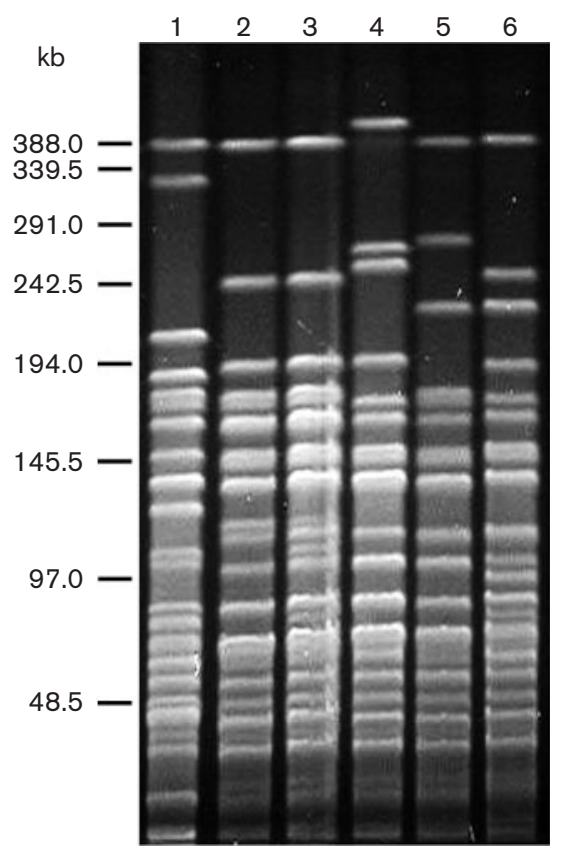

Fig. 5. PFGE profiles generated with Notl-digested genomic DNA obtained from $V$. cholerae $\mathrm{O} 1$ strains. The V. cholerae $\mathrm{O} 1$ strains included were classical 569B (lane 1), 1992 Kolkata variant VC51 and VC53 (lanes 2, 3), 2004 Mozambique variant B33 (lane 4), El Tor reference strain N16961 (lane 5) and 2003 Kolkata strain CO366 (lane 6). The band positions of the PFGE $\lambda$ ladder are indicated. 


\section{Conclusion}

Detailed analysis of 1992 V. cholerae variant O1 strains VC51 and VC53 revealed almost complete identity to the 2004 Mozambique variant $\mathrm{O} 1$ strains and is a testimony that the variant strains existed in Kolkata during 1992. Considering the time span between the isolation periods of Kolkata variant strains to that of the Mozambique variant strains, there is an immense possibility that Mozambique variants acquired certain genetic alterations, as evident in the PFGE.

The overall analysis indicated that 1992 Kolkata variant O1 strains VC51 and VC53 were built on the El Tor-like backbone but typically acquired the classical CTX prophage. Such genetic reassortment between the classical and El Tor traits represents genetic variations. It is well recognized that genetic replacement between divergent bacterial lineages can provide certain strains with an increased fitness in the environment or in the host. It is known that the El Tor biotype is more infective and sustainable but less virulent than the classical biotypes (Kaper et al., 1995). Therefore, the El Tor $V$. cholerae that produces the classical cholera toxin appears to be an altered strain that emerged causing a more severe disease. The year 1992 is a landmark in the history of cholera as O139 vibrios emerged and subsequently caused large epidemics. Since the emergence of O139 strains in late 1992, isolation of O1 El Tor vibrios from cholera cases has been virtually non-existent. However, a resurgence of $\mathrm{O} 1$ vibrios occurred within a span of a year and both serogroups co-existed for a long period of time. Interestingly, the resurgent $\mathrm{O} 1$ vibrios in the post-O139 era were reported to belong to newer clones as compared to the pre-O139 era (Sharma et al., 1997). To date, the factors that played a crucial role in the disappearance of pre-O139 O1 clones are incomprehensible. Retrospective analysis of the $\mathrm{O} 1$ strains indicated that biotype variants of $V$. cholerae $\mathrm{O} 1$ existed in Kolkata during 1992. Most probably the variant disappeared with the epidemic caused by the O139 vibrios. Our analysis of 1992 Kolkata variant O1 strains indicated that they were progenitor strains of the 2004 Mozambique variant O1 strains. Therefore, we presume that the 2004 Mozambique variant $\mathrm{O} 1$ strains of $V$. cholerae are a case of resurgence of certain clones of the $\mathrm{O} 1$ strains that were isolated in Kolkata during 1992.

\section{ACKNOWLEDGEMENTS}

This work was supported in part by the Council of Scientific and Industrial Research (CSIR), Government of India (no 37/1206/04/ EMR II), the Japan International Co-operation Agency (JICA/NICED project 054-1061-E-O), the Ministry of Health Labor and Family Welfare of Japan (project H17-Shinkou-3) and the Program of Founding Research Centers for Emerging and Reemerging Infectious Diseases, Ministry of Education, Culture, Sports, Science and Technology, Japan. T. P., K. G. and A. R. are the recipients of Senior Research Fellowships from the Department of Biotechnology (DBT) (project no. BT/PR6918/BRB/10/454/2005), the University Grant Commission (UGC), and the Indian Council of Medical Research (ICMR), Government of India, respectively.

\section{REFERENCES}

Ansaruzzaman, M., Bhuiyan, N. A., Nair, B. G., Sack, D. A., Lucas, M., Deen, J. L., Ampuero, J. \& Chaignat, C. L. (2004). Cholera in Mozambique, variant of Vibrio cholerae. Emerg Infect Dis 10, 20572059.

Ansaruzzaman, M., Bhuiyan, N. A., Safa, A., Sultana, M., McUamule, A., Mondlane, C., Wang, X. Y., Deen, J. L., von Seidlein, L. \& other authors (2007). Genetic diversity of El Tor strains of Vibrio cholerae O1 with hybrid traits isolated from Bangladesh and Mozambique. Int J Med Microbiol 297, 443-449.

Barrett, T. J. \& Blake, P. A. (1981). Epidemiological usefulness of changes in hemolytic activity of Vibrio cholerae biotype El Tor during the seventh pandemic. J Clin Microbiol 13, 126-129.

Bhattacharya, T., Chatterjee, S., Maiti, D., Bhadra, R. K., Takeda, Y., Nair, G. B. \& Nandy, R. K. (2006). Molecular analysis of the $r s t R$ and orfU genes of the CTX prophages integrated in the small chromosomes of environmental Vibrio cholerae non-O1, non-O139 strains. Environ Microbiol 8, 526-534.

Bik, E. M., Gouw, R. D. \& Mooi, F. R. (1996). DNA fingerprinting of Vibrio cholerae strains with a novel insertion sequence element: a tool to identify epidemic strains. J Clin Microbiol 34, 1453-1461.

Brosius, J., Ullrich, A., Raker, M. A., Gray, A., Dull, T. J., Gutell, R. R. \& Noller, H. F. (1981). Construction and fine mapping of recombinant plasmids containing the $r r n B$ ribosomal RNA operon of E. coli. Plasmid 6, 112-118.

Chakrabarti, A. K., Ghosh, A. N., Nair, G. B., Niyogi, S. K., Bhattacharya, S. K. \& Sarkar, B. L. (2000). Development and evaluation of a phage typing scheme for Vibrio cholerae O139. J Clin Microbiol 38, 44-49.

Chatterjee, S., Ghosh, K., Raychoudhuri, A., Pan, A., Bhattacharya, M. K., Mukhopadhyay, A. K., Ramamurthy, T., Bhattacharya, S. K. \& Nandy, R. K. (2007). Phenotypic and genotypic traits and epidemiological implication of Vibrio cholerae $\mathrm{O} 1$ and $\mathrm{O} 139$ strains in India during 2003. J Med Microbiol 56, 824-832.

Das, B., Halder, K., Pal, P. \& Bhadra, R. K. (2007). Small chromosomal integration site of classical CTX prophage in Mozambique Vibrio cholerae O1 biotype El Tor strain. Arch Microbiol 188, 677-683.

Davis, B. M., Kimsey, H. H., Chang, W. \& Waldor, M. K. (1999). The Vibrio cholerae $\mathrm{O} 139$ Calcutta bacteriophage $\operatorname{CTX} \varphi$ is infectious and encodes a novel repressor. J Bacteriol 181, 6779-6787.

Davis, B. M., Moyer, K. E., Boyd, E. F. \& Waldor, M. K. (2000). CTX prophages in classical biotype Vibrio cholerae: functional phage genes but dysfunctional phage genomes. J Bacteriol 182, 6992-6998.

Faruque, S. M., Tam, V. C., Chowdhury, N., Diraphat, P., Dziejman, M., Heidelberg, J. F., Clemens, J. D., Mekalanos, J. J. \& Nair, G. B. (2007). Genomic analysis of the Mozambique strain of Vibrio cholerae O1 reveals the origin of El Tor strains carrying classical CTX prophage. Proc Natl Acad Sci U S A 104, 5151-5156.

Kaper, J. B., Morris, J. G., Jr \& Levine, M. M. (1995). Cholera. Clin Microbiol Rev 8, 48-86.

Keasler, S. P. \& Hall, R. H. (1993). Detecting and biotyping Vibrio cholerae $\mathrm{O} 1$ with multiplex polymerase chain reaction. Lancet $\mathbf{3 4 1}$, 1661.

Kelly, M. T., Hickman-Brenner, F. W. \& Farmer, J. J., III (1992). Vibrio. In Manual of Clinical Microbiology, pp. 384-395. Edited by A. Balows, W. J. Hausler, Jr, K. L. Herrmann, H. D. Isenberg \& H. J. Shadomy. Washington, DC: American Society for Microbiology.

Kimsey, H. H. \& Waldor, M. K. (1998). СTX $\varphi$ immunity: application in the development of cholera vaccines. Proc Natl Acad Sci U S A 95, 7035-7039. 
Maiti, D., Das, B., Saha, A., Nandy, R. K., Nair, G. B. \& Bhadra, R. K. (2006). Genetic organization of pre-CTX and CTX prophages in the genome of an environmental Vibrio cholerae non-O1, non-O139 strain. Microbiology 152, 3633-3641.

Murray, M. G. \& Thompson, W. F. (1980). Rapid isolation of high molecular weight plant DNA. Nucleic Acids Res 8, 4321-4325.

Nair, G. B., Faruque, S. M., Bhuiyan, N. A., Kamruzzaman, M., Siddique, A. K. \& Sack, D. A. (2002). New variants of Vibrio cholerae O1 biotype El Tor with attributes of the classical biotype from hospitalized patients with acute diarrhea in Bangladesh. J Clin Microbiol 40, 3296-3299.

Olsvik, O., Wahlberg, J., Petterson, B., Uhlen, M., Popovic, T., Wachsmuth, I. K. \& Fields, P. I. (1993). Use of automated sequencing of polymerase chain reaction-generated amplicons to identify three types of cholera toxin subunit B in Vibrio cholerae O1 strains. J Clin Microbiol 31, 22-25.

Popovic, T., Fields, P. I. \& Olsvik, O. (1994). Detection of cholera toxin genes. In Vibrio cholerae and Cholera: Molecular to Global
Perspectives, pp. 41-52. Edited by I. K. Wachsmuth, P. A. Blake \& O. Olsvik. Washington, DC: American Society for Microbiology.

Rubin, E. J., Lin, W., Mekalanos, J. J. \& Waldor, M. K. (1998). Replication and integration of a Vibrio cholerae cryptic plasmid linked to the CTX prophage. Mol Microbiol 28, 1247-1254.

Safa, A., Bhuyian, N. A., Nusrin, S., Ansaruzzaman, M., Alam, M., Hamabata, T., Takeda, Y., Sack, D. A. \& Nair, G. B. (2006). Genetic characteristics of Matlab variants of Vibrio cholerae O1 that are hybrids between classical and El Tor biotypes. J Med Microbiol 55, 1563-1569.

Sharma, C., Nair, G. B., Mukhopadhyay, A. K., Bhattacharya, S. K., Ghosh, R. K. \& Ghosh, A. (1997). Molecular characterization of Vibrio cholerae O1 biotype El Tor strains isolated between 1992 and 1995 in Calcutta, India: evidence for the emergence of a new clone of the El Tor biotype. J Infect Dis 175, 1134-1141.

Trucksis, M., Michalski, J., Deng, Y. K. \& Kaper, J. B. (1998). The Vibrio cholerae genome contains two unique circular chromosomes. Proc Natl Acad Sci U S A 95, 14464-14469. 